\title{
La imagen de la juventud en la publicidad televisiva de Perú
}

\author{
The image of youth in television advertising from Peru
}

Silvia Liseti Quinte Rodríguez'

Rubén Darío Alania Contreras ${ }^{2}$

Víctor Ricardo Flores Rivas ${ }^{3}$

RECIBIDO: OCTUBRE 282019

ACEPTADO: NOVIEMBRE 132019

1 Licenciada en Ciencias de la Comunicación, docente, Universidad Continental, squinte@ continental.edu.pe, https://orcid.org/0000-0001-7359-0441

2 Licenciado en Ciencias de la Comunicación, Psicólogo, Doctor en Educación, docente, Universidad Continental, ralania@continental.edu.pe, https://orcid.org/0000-0003-4303-1037 3 Licenciado en Ciencias de la Comunicación, Maestro en Docencia Universitaria, Universidad Continental, vflores@continental.edu.pe, https://orcid.org/0000-0002-0243-2267 


\section{Resumen}

La investigación tuvo como objetivo caracterizar la imagen de la juventud mostrada en la publicidad televisiva del Perú, definiendo qué papel se asigna a los jóvenes en las piezas publicitarias de la televisión peruana. El estudio, de tipo básico y diseño descriptivo simple, tomó una muestra de 100 spots publicitarios, emitidos durante los meses de julio, agosto y setiembre de 2019. Se aplicó como instrumento de recolección de datos, la ficha de análisis de contenido de imagen de la juventud, diseñada para la investigación. Se halló que, predominantemente, se muestra a jóvenes de fenotipo caucásico, atlético y que destacan por su atractivo físico, con roles de belleza, cuidado del cuerpo y la propia imagen, teniendo el placer como objetivo principal. Se concluye que la imagen de la juventud en la publicidad televisiva nacional es discriminativa en el fenotipo que representa; superficial y hedonista, en los roles asignados, y homogenizante, en preferencias juveniles.

Palabras clave: juventud, imagen, estereotipo, publicidad, televisión

\section{Abstract}

The research aimed to characterize the image of youth shown in television advertising from Peru, defining the role assigned to young people in the advertising pieces of Peruvian television. The study, of basic type and simple descriptive design, took a sample of 100 advertising spots, broadcast during the months of July, August and September 2019. A youth image content analysis sheet, designed for the study, was applied as a data collection instrument. It was found that, predominantly, advertising shows young people of Caucasian and athletic phenotype and that stand out for their physical attractiveness, with roles of beauty, body care and the image itself, having pleasure as the main objective. It is concluded that the image of youth in national television advertising is discriminatory in the phenotype it represents; superficial and hedonistic, in the assigned roles, and homogenizing, in youth preferences.

Keywors: youth, image, stereotype, advertising, television 


\section{Introducción}

En los últimos decenios, se han multiplicado las investigaciones denominadas "de efectos", que se proponen determinar el alcance de la influencia que los mensajes mediáticos ejercen sobre la audiencia. Así, Emanuelli (2000) refiere que "la 'juvenilización' de la pantalla coincide ciertamente con un avance del 'efecto joven' que se relaciona con las formas de trabajo, pero también con los valores imperantes y evidentes en los procesos sociales y culturales materializados en la vida cotidiana" (p. 6).

De acuerdo con Emanuelli (2001), la imagen de juventud que presenta la televisión se basa en el modelo medio estadounidense, que a raíz de la globalización y la desregularización económica ha logrado un alcance internacional y tiende a homogeneizar gustos y consumos sociales y culturales.

La juventud, en la actualidad, ha dejado de ser una etapa meramente biológica para convertirse en una categoría social y cultural, cuya identidad es moldeada y exaltada constantemente por los medios de comunicación, imponiendo iconos fuera de contexto, que, sin embargo, cobran cada vez mayor protagonismo en la construcción de la identidad y aspiraciones de los jóvenes. Ya no son la edad ni los atributos físicos los que deciden de modo principal acerca de la condición juvenil o no de las personas. Los límites temporales que engloban la juventud se vuelven inciertos.

Según Macassi (2001), el término "culturas juveniles" se asocia a los modos de pensar, sentir, percibir y actuar que atraviesan las actividades de un grupo y los distinguen de otros, es decir, aquí sí se considera el espacio temporal y espacial, los cuales no eran considerados por las visiones etarias y de moratoria social desde al adultocentrismo.

Así también, Bringué, Navas y Sánchez (2005) sostienen que la instalación del adulto en la juventud no tiene que ver únicamente con la dimensión física, sino que afecta también a los estilos de vida; a cualquier adulto, sea cual sea su edad, le resulta fácil mantener la ilusión de la instalación permanente en la juventud. De ahí que sea sumamente complejo elaborar el perfil de la juventud en general, aunque, como los autores indican, una forma de hacerlo sería dividir el colectivo juvenil en diversos grupos o subgrupos de acuerdo al 
mayor número de criterios posibles distintos. Así, hoy en día se habla de una multiplicidad de subculturas juveniles.

Es en este contexto que se inscribe la presente investigación, la cual no se propone investigar efectos, sino que se fija en una fase anterior del proceso comunicativo: analiza los contenidos presentados en los medios televisivos. En concreto, el estudio buscó definir la imagen de la juventud presente en la publicidad televisiva del Perú.

\section{Método}

Por su propósito, la investigación es de tipo básico, nivel descriptivo y diseño no experimental, descriptivo simple.

La población estuvo conformada por los spots televisivos que presentan jóvenes, emitidos por las cadenas televisivas de cobertura nacional en el Perú, durante el trimestre de julio a setiembre de 2019. La muestra la constituyeron 100 piezas publicitarias, que fueron seleccionadas a través de un muestreo probabilístico.

Como técnica de recolección de datos se aplicó la observación sistematizada y como instrumento de recolección de datos, se empleó la Ficha de Análisis de la Imagen de la Juventud en la Publicidad Televisiva, diseñada para la investigación. La validez de contenido del instrumento se realizó mediante el juicio de cinco expertos y la confiabilidad de la misma a través de una muestra piloto de 20 spots televisivos internacionales, la cual fue sometida a la prueba de alfa de Cronbach, obteniendo una fiabilidad de 0.92. De este modo, el instrumento resultó válido y confiable.

El instrumento evalúa lasa dimensiones e indicadores propuestos por Bringué et al. (2005):

- Características formales de los anuncios.

- Análisis de algunos aspectos específicos de la juventud.

- La forma de vestir como exponente de un modo de ser.

- Roles y acciones de la juventud en la publicidad televisiva.

- Las diferencias de género. 


\section{Resultados}

El análisis de los spot publicitarios televisivos, se lograron los siguientes resultados.

\section{a. Características formales de los spots publicitarios}

Tabla 1

Papel de la persona joven en el anuncio

\begin{tabular}{lc}
\hline Papel asignado & Porcentaje \\
\hline Protagonista & 92 \\
\hline Aparición fugaz & 8 \\
\hline TOTAL & 100 \\
\hline
\end{tabular}

Tabla 2

Situación del joven en el anuncio

\begin{tabular}{lc}
\hline Situación & Porcentaje \\
\hline $\begin{array}{l}\text { Acompañado por jóvenes y } \\
\text { Otras personas }\end{array}$ & 40 \\
$\begin{array}{l}\text { Exclusivamente } \\
\text { acompañado por jóvenes }\end{array}$ & 43 \\
Otras situaciones & 7 \\
\hline TOTAL & 100 \\
\hline
\end{tabular}

Tabla 3

Tipos de productos anunciados en televisión

\begin{tabular}{lc}
\hline Tipo de productos & Porcentaje \\
\hline Bebidas alcohólicas & 14 \\
Bebidas no alcohólicas & 12 \\
Alimentación & 13 \\
Limpieza & 6 \\
Electrónica de consumo y & 5 \\
electrodomésticos & 2
\end{tabular}


Vehículos y accesorios

Confección, complementos y joyas

Finanzas, seguros y loterías

Telefonía y aparatos de

comunicación

Medios de comunicación,

publicaciones y artículos de oficina

Servicios públicos sin ánimo de lucro

Perfumería
7

5

9

12

4

3

5

Otros 3

TOTAL 100

Los datos previos proporcionan un panorama sobre los productos que utilizan en forma más recurrente la figura de la juventud como estrategia de promoción publicitaria. Como se aprecia en la tabla 3, hay una mayor presencia en los rubros de las bebidas alcohólicas y no alcohólicas, alimentos y telefonía y aparatos de comunicación.

\section{b. Análisis de algunos aspectos específicos de la juventud anunciada}

Tabla 4

Escenario en el que aparecen los jóvenes

\begin{tabular}{lcccc}
\hline Escenario & Mujer & Varón & Ambos & Total (\%) \\
\hline Doméstico & 12 & 4 & 8 & 25 \\
Lúdico & 6 & 4 & 9 & 19 \\
Laboral & 3 & 8 & 3 & 14 \\
Sin escenario & 5 & 5 & 3 & 13 \\
Calle & 4 & 4 & 2 & 10 \\
Deportivo & 4 & 2 & - & 6 \\
Educativo-cultural & - & 2 & - & 2 \\
Religioso-cultural & 1 & - & - & 1 \\
Otros & 2 & 4 & 4 & 10 \\
\hline Total & 37 & 33 & 30 & 100 \\
\hline
\end{tabular}


Como se observa en la tabla 4, el escenario del hogar es el que más se repite; este se entiende como un lugar de permanencia habitual de los jóvenes. En cuanto a la diferencia de género, se aprecia un predominio de estereotipos machistas, que sitúan en mayor porcentaje a la mujer en un contexto doméstico, y al varón, en un contexto laboral.

Tabla 5

Cualidades de los jóvenes

\begin{tabular}{lcccc}
\hline Cualidades & Mujer & Varón & Ambos & Total (\%) \\
\hline Alegre, divertido & 10 & 5 & 10 & 24 \\
Atractivo físico & 12 & 4 & 6 & 23 \\
$\begin{array}{l}\text { Nuevo, moderno } \\
\text { Sociable, amistoso, }\end{array}$ & 4 & 4 & 4 & 12 \\
$\begin{array}{l}\text { colaborador } \\
\text { Arriesgado, decidido }\end{array}$ & 3 & 4 & 3 & 10 \\
$\begin{array}{l}\text { Trabajador, profesional, } \\
\text { organizado, responsable }\end{array}$ & 1 & 4 & 2 & 7 \\
$\begin{array}{l}\text { Saludable, deportivo } \\
\text { Intelectual, educativo }\end{array}$ & 4 & 4 & - & 6 \\
$\begin{array}{l}\text { Bueno, bondadoso, } \\
\text { virtuoso }\end{array}$ & 1 & 2 & - & 6 \\
Triste, sufrido, agotado, & - & 3 & 2 & 5 \\
indiferente & - & 1 & 1 & 2 \\
\hline Total & 37 & 33 & 30 & 100 \\
\hline
\end{tabular}

La presentación de los jóvenes, mayoritariamente con cualidades de alegría y diversión, ratifican la característica desproblematizadora y evasiva de la publicidad. Se reafirma la idea de la juventud como una etapa de la vida despreocupada, en la que todavía no se han asumido responsabilidades y en la que primaría el elemento lúdico. Asimismo, la asociación del atractivo físico con la juventud obedece a la idealización de esta categoría, la cual provoca que los jóvenes se impongan cánones de belleza y que incluso lleguen a "esclavizarse" en su afán por mantener una imagen corporal acorde con estos. 
Tabla 6

Comportamiento hacia los demás

\begin{tabular}{lcccc}
\hline Comportamiento & Mujer & Varón & Ambos & Total (\%) \\
\hline No calificable* & 7 & 3 & 8 & 18 \\
Acuerdo & 12 & 8 & 6 & 12 \\
Distensión & 5 & 4 & 3 & 26 \\
Colaboración & 6 & 9 & 4 & 19 \\
Opinión y orientación & 4 & 4 & 3 & 11 \\
Solidaridad & 3 & 4 & 2 & 9 \\
Antagonismo & - & 1 & 2 & 3 \\
Tensión & - & - & 1 & 1 \\
Desacuerdo & - & - & 1 & 1 \\
\hline Total & 37 & 33 & 30 & 100 \\
\hline
\end{tabular}

* Es el caso de la persona joven que se halla sola o en medio de un grupo en el que no existe interacción.

En la tabla 6, se muestra que el ítem no calificable alcanza un alto porcentaje; esto indica que, en las piezas publicitarias, se recurre con frecuencia a la presencia en solitario del personaje joven; no aparece alguien hacia quien pueda orientar su comportamiento. Así también, el comportamiento más repetitivo de los jóvenes en la publicidad es la distención, que se refiere a la despreocupación, ausencia de tensión, soltura, falta de concentración. Otros tipos de comportamiento juvenil hacia los demás más frecuentes en los spots publicitarios son el de colaboración y el de acuerdo. 
Tabla 7

Imagen corporal de la juventud en la publicidad

\begin{tabular}{|c|c|c|c|}
\hline Aspectos & Mujer & Varón & Total (\%) \\
\hline \multicolumn{4}{|l|}{ Talla } \\
\hline Altos & 18 & 31 & 49 \\
\hline Medianos & 28 & 13 & 41 \\
\hline Bajos & 6 & 4 & 10 \\
\hline Total & 52 & 48 & 100 \\
\hline \multicolumn{4}{|l|}{ Contextura } \\
\hline Mesomorfos & 20 & 32 & 52 \\
\hline Ectomorfos & 29 & 8 & 37 \\
\hline Endomorfos & 3 & 8 & 11 \\
\hline Total & 52 & 48 & 100 \\
\hline \multicolumn{4}{|l|}{ Color de cabello } \\
\hline Cabello castaño & 25 & 15 & 40 \\
\hline Cabello negro & 13 & 24 & 37 \\
\hline Cabello rubio & 10 & 7 & 17 \\
\hline Pelirrojo & 4 & - & 4 \\
\hline Canoso & - & 2 & 2 \\
\hline Total & 52 & 48 & 100 \\
\hline \multicolumn{4}{|l|}{ Grupo étnico } \\
\hline Caucásico & 46 & 40 & 86 \\
\hline Mestizo & 4 & 6 & 10 \\
\hline Amerindio & - & 1 & 1 \\
\hline Negroide & 1 & 1 & 2 \\
\hline Oriental & 1 & - & 1 \\
\hline Total & 52 & 48 & 100 \\
\hline
\end{tabular}

Los aspectos físicos idealizados (ver tabla 7) mostrados por la publicidad televisiva reafirman la importancia y el valor que se le asigna a la imagen corporal en nuestra cultura. Sin embargo, los fenotipos raciales caucásicos, que más apariciones tienen en los spots analizados, no reflejan a la mayoría de la población peruana, de características más bien mestizas. Estas cifras 
ponen sobre el tapete las polémicas actitudes racistas que se le acusan a la publicidad en el Perú.

\section{c. La forma de vestir como exponente de un modo de ser}

Tabla 8

Vestuario de los jóvenes

\begin{tabular}{lccc}
\hline Tipos de vestuario & Mujer & Varón & Total (\%) \\
\hline Traje de calle & 22 & 33 & 55 \\
Traje de casa & 8 & 3 & 11 \\
Atuendo deportivo & 6 & 3 & 9 \\
Traje de fiesta o ceremonia & 6 & 3 & 9 \\
Traje de baño & 7 & 2 & 9 \\
Uniforme & 1 & 4 & 5 \\
Pijama & 2 & & 2 \\
\hline Total & 52 & 48 & 100 \\
\hline
\end{tabular}

Como se observa en la tabla 8, el tipo de vestuario que llevan los jóvenes con mayor frecuencia de apariciones es, ampliamente, el de calle. Esta elección está asociada a las características descritas antes, como la distención (comportamiento), las de alegre y divertido (cualidades) y el atractivo físico. No olvidemos que la publicidad construye a sus personajes como modelos que resumen una aspiración, es decir, crean prototipos para ser seguidos. 
Tabla 9

Estilo del vestuario de los jóvenes

\begin{tabular}{lccc}
\hline Estilos & Mujer & Varón & Total (\%) \\
\hline Deportivo & 18 & 20 & 38 \\
Elegante y serio & 16 & 14 & 30 \\
Profesional & 4 & 10 & 14 \\
Estrafalario & 2 & - & 2 \\
Otro estilo & 12 & 4 & 16 \\
\hline Total & 52 & 48 & 100 \\
\hline
\end{tabular}

En cuanto al estilo del vestuario, mostrado en la tabla 9, es el deportivo el de mayor número de apariciones. La publicidad vincula, de este modo, la ropa informal, cómoda, "de moda" con la juventud. Este estilo, denominado usualmente sport, también está asociado a las cualidades atribuidas a los jóvenes (alegría, distensión, diversión, entre otros); el estereotipo salta a la luz una vez más.

Por otro lado, el estilo elegante y serio, con una importante frecuencia de aparición, está relacionado con lo nuevo, moderno, incluso con lo profesional, pero siempre con una preponderancia del atractivo físico. Así, el "buen vestir" se suma al cuidado de la imagen corporal para construir el prototipo de joven creado por la publicidad.

\section{d. Roles y acciones de la juventud en la publicidad televisiva}

Tabla 10

Objetivos asignados a los jóvenes

\begin{tabular}{lccc}
\hline Objetivos & Mujer & Varón & Total (\%) \\
\hline $\begin{array}{l}\text { Belleza, cuidado del } \\
\text { cuerpo y de la propia } \\
\text { imagen }\end{array}$ & 26 & 8 & 34 \\
$\begin{array}{l}\text { Placer } \\
\text { Placer }\end{array}$ & 8 & 15 & 23
\end{tabular}




\begin{tabular}{lccc} 
Amistad & 8 & 3 & 11 \\
Éxito & 2 & 6 & 8 \\
Desarrollo personal & 3 & 6 & 9 \\
Satisfacción sexual & 1 & 8 & 9 \\
Familia & 3 & 1 & 4 \\
Fama & 1 & 1 & 2 \\
\hline Total & 52 & 48 & 100 \\
\hline
\end{tabular}

Las piezas publicitarias proyectan como uno de los principales objetivos de la persona joven, la belleza (ver tabla 10), que supone el cuidado del cuerpo y de la propia imagen. De esta manera, la publicidad televisiva peruana muestra a jóvenes centrados sobre todo en su propio yo. Respecto a la diferencia de género, se observa que esta aspiración de belleza es mayoritariamente femenina; otro estereotipo reflejado en los mensajes publicitarios.

El placer es otro de los objetivos asignados a los jóvenes. En general, este es un recurso permanente de la publicidad, pues vende la idea de que la persona alcanzará satisfacción personal a través del consumo de los bienes y servicios anunciados, o sea, cuando se convierta en consumidor.

Tabla 11

Roles de la juventud en la publicidad

\begin{tabular}{lccc}
\hline Roles & Mujer & Varón & Total (\%) \\
\hline Biodiferencial & 12 & 7 & 19 \\
$\begin{array}{l}\text { Supuestamente propio del } \\
\text { Sexo }\end{array}$ & 6 & 5 & 11 \\
Ocio y entretenimiento & 12 & 6 & 17 \\
Lúdico & 8 & 5 & 13 \\
Vida social & 3 & 7 & 10 \\
Consumidor & 3 & 6 & 9 \\
Trabajador & 2 & 6 & 8 \\
Familiar y doméstico & 4 & 1 & 5
\end{tabular}




\begin{tabular}{lccc} 
Solidario & 1 & 4 & 5 \\
$\begin{array}{l}\text { Relacionado con lo } \\
\text { educativo y cultural }\end{array}$ & 1 & 1 & 2 \\
\hline Total & 52 & 48 & 100 \\
\hline
\end{tabular}

Como se observa en la tabla 11, el principal rol asignado a los jóvenes por la publicidad es el biodiferencial, esto es, que en la asignación de roles y papeles prima el ser joven antes que el género concreto de los actores.

El segundo rol con más apariciones es el de ocio y entretenimiento. Para los jóvenes, contar con espacios y momentos para distraerse y divertirse es prácticamente una necesidad; además, son estas ocasiones las que le permiten interactuar y crear vínculos con gente de su misma edad y afinidades.

No es casualidad que en tercer lugar figure el rol lúdico, relacionado al anterior en cuanto a formas de satisfacer la necesidad de ocio y diversión. Del modo como la publicidad la concibe, esta necesidad puede ser satisfecha a través del consumo.

\section{Discusión}

En nuestra cultura actual, la importancia de la imagen corporal (aspecto físico) así como de la apariencia ("buen lucir") ha logrado una hegemonía nunca antes vista, y alcanza particularmente a los jóvenes, sobre todo, del sexo femenino. Así lo afirma también Figueiredo (2016), para quien la publicidad recurre, por lo general, a cuerpos que corresponden a un arquetipo estético, a una corporeidad supuestamente ideal, en detrimento de la diversidad; esto, enfatiza, sería reflejo de una sociedad que valora una apariencia estandarizada de los individuos. Además, como los resultados vistos antes indican, es en el género femenino en el que recae, la mayoría de veces, la idealización de la belleza. Al respecto, Figueiredo encontró que la perfección corporal femenina presentada por la publicidad encierra una trilogía: belleza, delgadez y juventud; atributo este último abordado en la presente investigación.

La imagen física constituye con frecuencia, para los jóvenes, un elemento 
importante, casi principal, de la identidad que están buscando o construyendo en esa etapa de su vida, y a pesar de las favorables condiciones naturales o de partida con las que se encuentran, lo exigente de los estereotipos vigentes se convierte, para muchos de ellos, en una fuente de tensión y sufrimiento. De ahí la advertencia de Bringué et al. (2005) respecto a la insistencia de la publicidad televisiva en lo juvenil y en el atractivo físico: al presentarse casi como una exigencia, puede convertirse en un elemento peligroso a la hora de configurar la personalidad. Asimismo, refieren, tanto las cualidades como los roles atribuidos a los jóvenes por la lógica publicitaria, si bien incluyen rasgos favorables, pueden generar insatisfacción si no se hace hincapié en presentar objetivos de mayor trascendencia y más accesibles.

En una sociedad de consumo, los jóvenes viven muy pendientes, casi esclavos, de su imagen corporal y de la ropa que "deben" lucir; esa es la razón de que, como señala Méndiz (2005), la juventud esté en el punto de mira de los publicistas. Según el autor, el target juvenil es considerado el más prometedor para las marcas consolidadas, pues constituyen "un público vulnerable, sensible a mensajes emotivos y escasamente racionales... además desarrolla fácilmente mecanismo de identificación con las marcas" (p. 105). Así, la publicidad genera el denominado culto de las marcas, cuya característica principal es la dependencia de aquellas que venden la promesa de un estatus, de un estilo de vida que distingue.

De otro lado, en el caso peruano en particular, es bastante discutible que los prototipos físicos impuestos por la publicidad televisiva, como aspiración estética hacia los jóvenes, estén extremadamente alejados de la realidad y no representen los rasgos físicos y étnicos del grueso de la población; entonces, se plantea la interrogante de si la publicidad es racista o es que solo refleja las aspiraciones de una sociedad racista. Al respecto, el estudio de Mendoza (2015) reveló que los creativos de las agencias publicitarias peruanas consideran que cambiar la estructuración de los anuncios incluyendo, por ejemplo, lo andino no sería prudente porque la mayoría de los consumidores (público) y clientes (empresas) continúan validando los modelos caucásicos. Las agencias, señala, buscan estar en "sintonía" con los imaginarios sociales, que aún conciben el fenotipo caucásico como el ideal, y mediante los anuncios que elaboran siguen reproduciéndolos. 
Además de la imagen y la apariencia, otro aspecto de la juventud que la publicidad muestra es su comportamiento. Como hemos revisado antes, se proyecta la idea del joven alegre, divertido, despreocupado, etc. En términos generales, se trata de cualidades que sí pueden estar asociadas al grupo etario correspondiente a dicha etapa del desarrollo; sin embargo, no dejan de constituir un estereotipo y, a la vez, un prejuicio que los propios jóvenes rechazarían, tal como Palacios (2017) sostiene. De acuerdo con la autora, los jóvenes reconocen en la narrativa publicitaria ciertos estereotipos, de los cuales muchos se toman por verdaderos sin un razonamiento previo, convirtiéndolos en prejuicios. Por ejemplo, considerar al joven como eternamente alegre, desenfrenado, "loco", aventurero y opuesto a la autoridad paterna, contradice otras visiones de los jóvenes como sujetos capaces de asumir actitudes distintas, no las "predecibles", de acuerdo a las circunstancias. Se observa, entonces, que la publicidad continúa no solo creando, sino también, reproduciendo estereotipos y prejuicios.

El discurso publicitario se basa en la difusión de sistemas de valores. Bringué et al. (2005) señalan que los valores pueden presentarse como un ideal que exige adhesión o manifestarse en conductas o cosas concretas. De este modo, la juventud constituye un valor en sí misma, pues no solo se circunscribe al aspecto de la edad, sino que hoy se ha convertido, y en gran parte por efecto de la publicidad, en un estilo. Méndiz (2005) la considera un "valor universal" para todos los rangos de edad. Lo que hace la publicidad es emplear las características asociadas a la juventud para vender cualquier tipo de producto.

\section{Conclusiones}

La publicidad televisiva en el Perú, representa a la juventud, como una categoría social idealizada, a la que asocia aspectos como el atractivo físico y estilo de vida hedonista.

La publicidad televisiva peruana, al idealizar fenotipos y estilos de vida alejados de la realidad racial o étnica del país, genera aspiraciones que pueden producir en los jóvenes, insatisfacción y confusión en la definición de su identidad y sentido social de pertenencia. 
La producción de anuncios publicitarios televisivos en el Perú, recurre constantemente a contenidos discriminatorios basados en estereotipos físicos, de género y clase, que resultan muy efectivos para el marketing y sociedad de consumo; pero no contribuyen a la igualdad de género, eliminación del racismo o inclusión social.

En cuanto al tratamiento de la juventud como valor, los spots publicitarios televisivos peruanos son desproblematizadores y superficiales, pues otorgan excesiva importancia al entretenimiento y ocio, estereotipando a los jóvenes, como grupos hedonistas con una incesante búsqueda de placer.

Existe una excesiva presentación del cuerpo femenino perfecto, junto con la exacerbación del aspecto sexual o la utilización de la mujer como un mero elemento decorativo.

\section{Referencias bibliográficas}

Bringué, X., Navas, A., y Sánchez, J. J. (2005). La imagen de la juventud en la publicidad televisiva (Informe). Navarra, España: Consejo Audiovisual de Navarra. Recuperado de http://dadun.unav.edu/ bitstream/10171/17124/1/InformeJuventud.pdf

Emanuelli, P. B. (2000). Juvenilización de la pantalla y consumo televisivo. Hacia la homogeneización de preferencias juveniles. $\vee$ Congreso Latinoamericano de Ciencias de la Comunicación, Santiago de Chile.

Emanuelli, P. B. (2001). Posmodernidad y globalización en los medios masivos de comunicación. La TV por cable en Córdoba, Argentina: ¿̀hacia la homogeneización de identidades culturales? Revista Latina de Comunicación Social, 4(39). Recuperado de https://www.redalyc.org/ pdf/819/81939405.pdf

Figueiredo, C. (2016). Beleza, magreza e juventude: a perfeição corporal feminina na publicidade e a corporeidade disruptiva da Dove. Comunicación, 35, 13-27. Recuperado de https://revistas.upb.edu.co/ index.php/comunicacion/article/view/7096/6488

Macassi, S. (2001). Culturas juveniles, medios y ciudadanía: el nuevo horizonte 
generacional y las disyuntivas de la inserción de los jóvenes en la sociedad. Lima: Calandria.

Méndiz, A. (2005). La juventud en la publicidad. Revista de Estudios de Juventud, 68, 104-114. Recuperado de http://www.injuve.es/sites/default/files/ revista68_9.pdf

Mendoza, H.J. (2015). Racismo complaciente: (I) Lógicas delasrepresentaciones sociales de los peruanos desde la agencia publicitaria (Tesis de maestría). Pontificia Universidad Católica del Perú, Lima. Recuperada de http:// tesis.pucp.edu.pe/repositorio/handle/20.500.12404/6691

Palacios, J. A. (2017). Jóvenes y publicidad. Estereotipos, prejuicios, invisibilización y adultocentrismo. Revista Luciérnaga-Comunicación, 9(17), 1-11. Recuperado de https://dialnet.unirioja.es/descarga/ articulo/6563561.pdf 